\title{
QUANTUM INSPIRED EVOLUTIONARY ALGORITHM FOR SOLVING MULTIPLE TRAVELLING SALESMAN PROBLEM
}

\author{
Bhagwan Swain ${ }^{1}$, Rajdeep Ghosh ${ }^{2}$ \\ ${ }^{1}$ Department of Information Technology, Assam University, India \\ ${ }^{2}$ Department of Information Technology, Assam University, India
}

\begin{abstract}
Quantum computing is a relatively new but very promising field of computer science. It provides an alternative way of building computers which are significantly better than current day's classical computers. Here in this paper, we attempt to develop an algorithm which makes use of the concepts of quantum computers but are actually run on classical computers. Hence this is rather a novel approach. The algorithm is actually an optimization algorithm for solving the much famous multiple travelling salesman problem (mtsp). The algorithm further merges the methodologies followed by evolutionary algorithms.
\end{abstract}

So, at first we model an overall algorithm based on these concepts. Our algorithm is termed as quantum inspired evolutionary algorithm. With this basic approach we use multi chromosome technique and update solution using particle swarm optimization technique. Later on we compare these results with standard optimal solution for the problem and present a comparison for the same.

Keywords: Quantum inspired algorithm, Evolutionary Algorithm, Multi-Chromosome Technique, Q-bit, QEA. ***

\section{INTRODUCTION}

Quantum computing is a new field in computer science which has induced intensive investigations and researches during the last decade. It takes its origins from the foundations of the quantum physics. The parallelism that the quantum computing provides reduces obviously the algorithmic complexity. Such an ability of parallel processing can be used to solve efficiently optimization problems.Since there are no powerful quantum machines till today,some ideas such as simulating quantum algorithms on conventional computers or combining them to existing methods have been suggested to get benefit from this new science. In this paper we are using a combination of evolutionary algorithms and quantum computing principles which has already proved its usefulness in solving many problems such as the knapsack problem,multiobjecive image segmentation etc. The proposed approach use quantum bit representation instead of classical bits and use the evolutionary algorithm in addition with particle swarm optimization for obtaining an optimal solution for multi-travelling salesman problem.

\section{TRAVELLING SALESMAN PROBLEM (TSP)}

The travelling salesman problem (TSP) is an NP-hard problem in combinatorial optimization studied in operations research and theoretical computer science. The traveling salesman problem (TSP) was studied in the 18th century by a mathematician from Ireland named Sir William Rowam Hamilton and by the British mathematician named Thomas Penyngton Kirkman. Detailed discussion about the work of Hamilton \& Kirkman can be seen from the book titled Graph Theory (Biggs et al. 1976). [1]

\subsection{Definition}

Given a set of cities and the cost of travel (or distance) between each possible pairs, the TSP, is to find the best possible way of visiting all the cities and returning to the starting point that minimize the travel cost (or travel distance).

\subsection{Complexity}

Given $\mathrm{n}$ is the number of cities to be visited, the total number of possible routes covering all cities can be given as a set of feasible solutions of the TSP and is given as (n-1)!/2.

\subsection{MTSP}

The $\mathbf{m T S P}$ is defined as: In a given set of nodes, let there are $\mathrm{m}$ salesmen located at a single depot node. The remaining nodes (cities) that are to be visited are intermediate nodes. Then, the mTSP consists of finding tours for all $\mathrm{m}$ salesmen, who all start and end at the same or multiple depot, such that each intermediate node is visited exactly once and the total cost of visiting all nodes is minimized. Suppose that the number of cities is $\mathrm{n}$ and $\mathrm{m}$ is the number of salesman, then $\mathrm{m} \ll \mathrm{n}$ and $\mathrm{m}$ and $\mathrm{n}$ are all discrete numbers.[1][2]

Mathematically, The mTSP is defined on a graph $G=(V, A)$, where $\mathrm{V}$ is the set of $\mathrm{n}$ nodes (vertices) and $\mathrm{A}$ is the set of arcs (edges). Let $C=(c i j)$ be a cost (distance) matrix associated with 
A. The matrix $\mathrm{C}$ is said to be symmetric when $c i j=c j i, \forall i, j \in A$ and asymmetric otherwise. If $c i j \geq c j k+c i k,, \forall i, j, k \in V, C$ is said to satisfy the triangle inequality. The cost metric can be defined in terms of distance, time, etc.

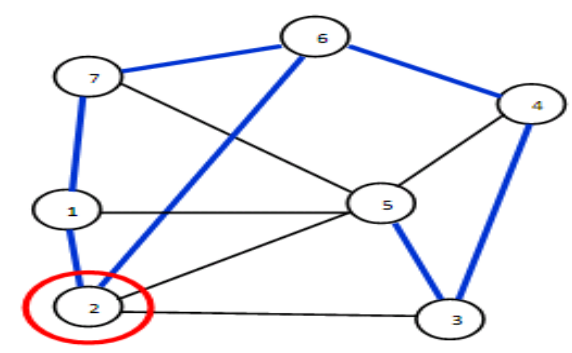

Fig1: Showing a graph for TSP

\section{BASIC CONCEPTS}

\subsection{Quantum Computing}

Quantum inspired computing is characterised by:

- The use of 'quantum-inspired' computational methods which is based on some principles of quantum mechanics such as q-bits, interference, standing waves, coherence etc.

- The use of classical algorithm for checking whether the solution generated by the quantum-inspired algorithm is correct .[5]

\subsubsection{Q-bits}

In classical computers there are two bits representing data. But a quantum computer maintains Q-bits. Q-bit has a quaternary nature. TheQ-bit is the quantum analogue of the bit, the classical fundamental unit of information. Classical bits can be either in 0 or 1 state. But Q-bits can be in two states denoted by $|0\rangle$ or $|1\rangle$ or a linear superposition of these two states. The notation |\rangle is called a state, a vector or a ket. A Q-bit is a bit of information that can be both zero and one simultaneously. A Q-byte is made up of eight Q-bits and can have all values from zero to 255 simultaneously. In general the physical state of a Q-bit is the superposition of two states and is given by: $|\boldsymbol{\psi}\rangle=\boldsymbol{\alpha}|\mathbf{0}\rangle+\boldsymbol{\beta}|\mathbf{1}\rangle$ Where, $|\boldsymbol{\psi}\rangle$ is the superposition state. $\boldsymbol{\alpha}, \boldsymbol{\beta}$ are complex numbers. $|\boldsymbol{\alpha}|^{2}$ represents the probability of finding $|\boldsymbol{\psi}\rangle$ in state 0 . $|\boldsymbol{\beta}|^{2}$ represents the probability of finding $|\psi\rangle$ in state 1 . This formalism for a quantum bit is a direct extension of one way to describe a classical computer. That is, way may write that a classical bit $|\omega\rangle$ is in the state $\boldsymbol{\alpha}|\mathbf{0}\rangle+\boldsymbol{\beta}|\mathbf{1}\rangle$. The only difference is $\alpha$ and $\beta$ are defined not over the complex numbers but rather from the set $\{0,1\}$. That is $\{\alpha, \beta\} \in\{0,1\}$.

\subsection{Genetic Algorithms}

Genetic algorithms derive from the evolution theory. They were introduced in 1975 by John Holland and his team as a highly parallel search algorithmIn genetic algorithms, this principle is traduced into the problem of finding the best individuals represented by chromosomes.

So, each chromosome encodes a possible solution for the given problem and, starting from a population of chromosomes, the evolution process performs a parallel search through the solutions' space. The fitness is measured for each individual by a function related to the objective function of the problem to be solved. Basically, a genetic algorithm consists of three major operations: selection, crossover, and mutation. The selection evaluates each individual and keeps only the fittest ones in the population. In addition to those fittest individuals, some less fit ones could be selected according to a small probability. The others are removed from the current population. The crossover recombines two individuals to have new ones which might be better. The mutation operator induces changes in a small number of chromosomes units. Its purpose is to maintain the population diversified enough during the optimization process. [6][7]

\subsection{Particle Swarm Optimisation}

PSO was formulated by Edward and Kennedy in 1995. The thought process behind the algorithm was inspired by the social behavior of animals, such as bird flocking or fish schooling. PSO is similar to the continuous GA in that it begins with a random population matrix. Unlike the GA, PSO has no evolution operators such as crossover and mutation. The rows in the matrix are called particles (same as the GA chromosome). They contain the variable values and are not binary encoded. Each particle moves about the cost surface with a velocity .The particles update their velocities and positions based on the local and global best solutions:

$$
\begin{aligned}
& v_{m, n}^{\text {naw }}=v_{m, n}^{\text {old }}+\Gamma_{1} * r_{1} x\left(p_{m, n}^{\text {local best }}-p_{m, n}^{\text {old }}\right)+\Gamma_{2} * \\
& \eta_{2} x\left(p_{m, n}^{\text {global best }}-p_{m, n}^{\text {old }}\right)
\end{aligned}
$$

$p_{m n}^{\text {new }}=\left(p_{m n}^{\text {old }}+v_{m n}^{\text {new }}\right) \quad$ where, $v_{m, n}=$ particle velocity $p_{m, n}=$ particle variables. $r_{1}, r_{2}=$ independent + uniform random numbers, $\Gamma_{1} \Gamma_{2}=$ learning factors $=2$.

$$
\begin{gathered}
p_{m, n}^{\text {global best }}=\text { best global solution } \\
p_{m, n}^{\text {local best }}=\text { best local solution }
\end{gathered}
$$

The PSO algorithm updates the velocity vector for each particle then adds that velocity to the particle position or values. Velocity updates are influenced by both the best global solution associated with the lowest cost ever found by a particle and the best local solution associated with the lowest cost in the present population.[4] 


\subsection{Quantum Evolutionary Algorithm (QEA):}

They can be classified into two fields. One concentrates on generating new quantum algorithms using automatic programming techniques such as genetic programming. The other concentrates on quantum-inspired evolutionary computing for a classical computer, a branch of study on evolutionary computing that is characterized by certain principles of interference, coherence, superposition etc. A Q-bit individual is defined by a string of Q-bits. The Q-bit individual has the advantage that it can represent a linear superposition of states (binary solutions) in search space probabilistically. Thus, the Qbit representation has a better characteristic of population diversity than other representations.[3]

\subsubsection{Quantum Individual Repre-Sentation}

A Q-bit is defined as the smallest unit of information. A Q-bit individual is defined as a string of Q-bits.

$$
\mathrm{Q}(\mathrm{t})=\left\{\mathrm{q}_{1}^{\mathrm{t}}, \mathrm{q}_{2}^{\mathrm{t}}, \ldots \ldots, \mathrm{q}_{\mathrm{n}}^{\mathrm{t}}\right\}
$$

at generation $t$,where, $\mathrm{n}$ is the population size, $\mathrm{q}_{\mathrm{j}}^{\mathrm{t}}, \mathrm{j}=1,2, \ldots, \mathrm{n}$ , is each Q-bit individual.Since the Q-bit representation is able to express as a linear superposition of states probabilistically, it is profitable for generating diversity in the evolutionary process.

A Q-bit individual is defined as:

$q_{j}^{t}=\left[\begin{array}{l|l}\alpha_{1}^{t} & \alpha_{2}^{t} \\ \beta_{1}^{t} & \beta_{2}^{t}\end{array}|\cdots| \begin{array}{l}\alpha_{m}^{t} \\ \beta_{m}^{t}\end{array}\right]$

Where, $\mathrm{m}$ is the number of Q-bits, and i.e., the string length of the Q-bit individual, and $\mathrm{j}=1,2 \ldots \mathrm{n}$ and $\left|\boldsymbol{\alpha}_{\mathrm{j}}^{\mathbf{t}}\right|^{\mathbf{2}}+\left|\boldsymbol{\beta}_{\mathrm{j}}^{\mathbf{t}}\right|^{\mathbf{2}}=1$. Initially, QEA can represent diverse individuals probabilistically because a Q-bit individual represents the linear superposition of all possible states with the same probability. As the probability of each Q-bit approaches either $|1\rangle$ or $|0\rangle$ by the Q-gate, the Qbit individual converges to a single state and diversity property disappears gradually.

\subsubsection{Structure of QEA}

Procedure QEA

Begin
$t \leftarrow 0$
initialize $Q(t)$
make $P(t)$ by observing the states of $Q(t)$
repair $P(t)$
evaluate $f\left(X_{j}^{0}\right)$
store the best solutions among $P(t)$ into $B(t)$
While (not termination condition) do
$\quad$ Begin
$\quad t \leftarrow t+1$
make $P(t)$ by observing the states of $Q(t-1)$
evaluate $f\left(X_{j}^{t}\right)$
update $Q(t)$ using $Q$-gate

store the best solutions among $P(t)$ into $B^{t}$ and $f\left(B^{t}\right)$

End

End

Where, $Q(t)=\left\{q^{t}{ }_{1}, q^{t}{ }_{2}, q^{t}{ }_{3}, \ldots q^{t}{ }_{n}\right\} ; P(t)=\left\{X^{t}{ }_{1}, X^{t_{2}}, X^{t_{3} \ldots \ldots . .} X^{t}{ }_{n}\right\} ; B^{t}$ $\epsilon X_{j} ; X_{j}^{t}=\left\{X^{t}{ }_{1 j}, X^{t}{ }_{2 j}, X_{3}{ }_{3} \ldots \ldots . . X^{t}{ }_{j m}\right\} ; \quad i=1,2 \ldots . . m ; j=1,2 \ldots n$. In the step of "initialize $\boldsymbol{Q}(\boldsymbol{t})$ " at $\mathrm{t}=0$ of all $\mathrm{q}_{\mathrm{j}}{ }^{0}$ in $\mathrm{Q}(0)$ are initialized with random probability. It means that in each $\mathrm{m} Q$ bits, $\mathrm{q}_{\mathrm{j}}^{0}$ represents the linear superposition of all possible states with the same probability. To obtain the binary string, the step of 'make $\boldsymbol{P}(\boldsymbol{t})$ by observing the state of $\mathrm{Q}(\mathrm{t})$ ' can be implemented for each Q-bit individual as follows. When observing the state of $\mathrm{Q}(\mathrm{t})$, the value $\mathrm{x}_{\mathrm{ji}}^{\mathrm{t}}=0$ or 1 of $\mathrm{P}(\mathrm{t})$ is determined by the probability $\left|\boldsymbol{\alpha}_{\mathrm{ji}}^{\mathbf{t}}\right|^{\mathbf{2}}$ or $\left|\boldsymbol{\beta}_{\mathrm{ji}}^{\mathbf{t}}\right|^{\mathbf{2}}$. The steps of 'repair ( $\boldsymbol{t})$ ' and 'evaluate $\boldsymbol{f}\left(\boldsymbol{X}_{j}^{t}\right)$ ' are according to the problems, where $\mathrm{f}(\mathrm{X})$ is the fitness function. The update procedure is problem specific. That can be done by using Q-gate, Particle Swarm Optimization, or Ant Colony Optimization etc.

\section{SOLUTION APPROACH}

A solution procedure for designing a quantum inspired evolutionary algorithm for multi travelling salesman problem by multi-chromosome techniques is as follows: Here all the individuals of the population are represented by as set of chromosomes each representing a possible solution to a problem. The number of chromosomes $m$ present in an individual represents the number of salesman and each gene or the positive integer in a chromosome represents a city value. The sum of all the city values is equal to the $n$ randomly generated cities.In each generation new sets of individuals are formed from the existing sets using the genetic procedures crossover and mutation.

The cost of all the individuals in each generation is calculated and are stored which are compared with the cost of individuals of other generations. The lowest cost thus obtained gives the best resulting tour.

\section{Procedure for initialization of salesman part Begin $t \leftarrow 0$ \\ Initialize $Q_{s}(t)$ \\ Make $P_{s}(t)$ by observing the states of $Q_{s}(t)$ Repair $P_{s}(t)$ \\ End}

\begin{tabular}{l}
\hline Procedure for generation of city sequence part Begin \\
\hline$t^{\prime} \leftarrow 0$ \\
$\quad$ initialize $Q_{c}\left(t^{\prime}\right)$ \\
$\quad$ make $P_{c}\left(t^{\prime}\right)$ \\
$\quad$ repair $P_{c}\left(t^{\prime}\right)$ \\
$\quad$ evaluate the cost of individuals \\
$\quad$ initialize gbest \\
While (not termination condition) do \{ \\
$t^{\prime} \leftarrow t^{\prime}+1$ \\
perform crossover $Q_{c}\left(t^{\prime}\right)$ \\
perform mutation $Q_{c}\left(t^{\prime}\right)$
\end{tabular}


update state using update $Q_{c}\left(t^{\prime}\right)$

make $P_{c}\left(t^{\prime}\right)$ by observing the states of $Q_{c}\left(t^{\prime}\right)$

repair $P_{c}\left(t^{\prime}\right)$

evaluate the cost of individuals

store gbest

\}

End while

End

Where, $\boldsymbol{Q}_{s}(t)$ is the Q-bit individual for the number of cities per salesman. $\boldsymbol{Q}_{c}\left(\boldsymbol{t}^{\prime}\right)$ is the Q-bit individual for the assigned cities. $\boldsymbol{P}_{s}(t)$ is the measured Q-bit individual for the number of cities per salesman. $\mathrm{P}_{s}\left(\boldsymbol{t}^{\prime}\right)$ is the measured Q-bit individual for the assigned cities. Initialize $\mathbf{Q}(\mathbf{T})$ :In this procedure, at $\mathrm{t} \leftarrow 0$, the $1^{\text {st }}$ part of the chromosomes Qbit individuals, $q_{j}^{t}$ i.e. $\left[\begin{array}{l}\alpha_{j i}^{t} \\ \beta_{j i}^{t}\end{array}\right]$ are initialized with random probabilities assigned to each of them, such that $|\boldsymbol{\alpha}|^{2}+|\boldsymbol{\beta}|^{2}=\mathbf{1}$.It means that in each in each m-Q-bits, $\mathrm{q}_{\mathrm{j}}^{\mathrm{t}}$ represents the linear superposition of all possible states with the random probability. The Make $\mathbf{P}_{\mathbf{s}}(\mathbf{t})$ and $\mathbf{P}_{\mathbf{c}}(\mathbf{t})$ can be implemented for each Q-bit individual as follows. When observing the state of $\mathrm{Q}(\mathrm{t})$, the value $\mathrm{x}_{\mathrm{j}}^{\mathrm{t}}=0$ or 1 of $\mathrm{P}(\mathrm{t})$ can be determined by the probability of $\left|\boldsymbol{\alpha}_{j i}^{t}\right|^{2}$ or $\left|\boldsymbol{\beta}_{j i}^{t}\right|^{2}$ as follows : This step is same for both $P_{s}(t)$ and $P_{c}(t)$. After generating the binary string, corresponding decimal value is obtained by converting the binary string into decimal values whereas the procedures repair $\mathbf{P}_{s}(t)$ and $\mathbf{P}_{c}(t)$ are used for repairing the matrices ina accordance with a specific criterion,the crossover $\mathbf{Q}_{\mathbf{c}}(\mathbf{t})$ and mutation $\mathbf{Q}_{\mathbf{c}}(\mathbf{t})$ are evolutionary operators used for performing crossover and mutation over the population of individuals and update $\mathbf{Q}_{\mathbf{c}}(\mathbf{t})$ uses particle swarm optimization to update the individuals of the population.

\section{RESULT AND COMPARISION}

The proposed algorithm is coded in Matlab and implemented on a intel core i3,2.4 GHZ,(2GB RAM), operation system is windows 7. the efficiency and performance of the proposed algorithm (PA) is compared with some of the best techniques designed including modified genetic Algorithm (MGA). These algorithms are applied and tested on several instances from TSP problemsavailable on the TSPLIB including Pr76, Pr152, Pr299 with 5 salesman.[6]

Table 1: Result and Comparision [6]

\begin{tabular}{|l|l|l|l|}
\hline Instances & MACO & $\begin{array}{l}\text { Best Known } \\
\text { Solution }\end{array}$ & Proposed \\
\hline pr76 & 178597 & 157444 & 167891 \\
\hline pr152 & 127839 & 127839 & 127839 \\
\hline Pr299 & 82106 & 82106 & 81950 \\
\hline
\end{tabular}

\section{REFERENCES}

[1]. Tolga Bektas, "Travelling Salesman Problem Formulation and solution", Omega 34 (2006) $209-219$

[2]. Bektas, T. (2006). "The multiple traveling salesman problems: an overview of formulations and solution procedures". OMEGA: The International Journal of Management Science, 34(3), 209-219.

[3]. Ge-Xiang Zhang “, A Quantum-Inspired Evolutionary Algorithm Based on $\mathrm{P}$ systems for Knapsack Problem, Fundamental Informatica" 87(2008), pp 1-24

[4]. Yan Wang, Xiao- Yue Feng, Yan-Xin Huang, Dong-Bing $\mathrm{Pu}$, Wen-Gang Zhou, Yan-Chun Liang, Chun- Guang Zhou, "A novelquantum swarm evolutionary algorithm and its applications, Neurocomputing "70, 2007, pp 633-640

[5]. Ajit Narayanan and Moore,"Quantum inspired genetic algorithm"1996

[6]. Mohammad Sedighpoura, Majid Yousefikhoshbakhtb,Narges Mahmoodi Daranic," An Effective Genetic Algorithm for Solving the Multiple Traveling Salesman Problem" Journal of Optimization in Industrial Engineering 8 (2011)

[7]. Arther E Carter, Design and Application of Genetic Algorithms for the Multiple Traveling Salesperson Assignment Problem, April 21, 2003.

[8]. Gohar Vahdati, Mehdi Yaghoubi, Mahdieh Poostchi, M.B. Naghibi, A new Approach to Solve Travelling Salesman Problem Using Genetic Algorithm Based on Heuristic Crossover and mutation Operator, 2009 International Conference of Soft Computing and Pattern Recognition, pp 1-5 